\title{
Gangrenous Sigmoid Volvulus - A Case Report with review of literature
}

\author{
Shekar Y Tati ${ }^{1}$, Ganapathi Puranik ${ }^{1}$, Chidananda $\mathrm{KV}^{1}$ Karthikeyan $\mathrm{K}^{2}$ Somdeep \\ Ghosh $^{2}$, Harris Mohammed $\mathrm{P}^{2}$, Akshay Pai ${ }^{2}$, Senthilnathan $\mathrm{K}^{2}$, Venkataramana \\ $\mathrm{G}^{2}$, Navya $\mathrm{BN}^{3}$
}

1Professor, 2Post graduate, department of general surgery. 3Assistant professor, department of pathology.KVG Medical College \& Hospital, Sullia- DK, Karnataka, India.

\begin{abstract}
Sigmoid volvulus is a life threatening surgical emergency which demands early surgical intervention. The condition is prevalent all over the world. Both sexes can be involved but more common in males. Mostly it is a disease of elderly males but can occur in neonates to young individuals. The condition presents in two distinct form gangrenous and non gangrenous sigmoid volvulus. Gangrenous volvulus is associated with high morbidity.

Volvulus patient presents with pain, distension of abdomen and absolute constipation. Plain X-ray erect abdomen will give us definite diagnosis. In doubtful cases double contrast barium enema will clinch the diagnosis. In non gangrenous sigmoid volvulus sigmoidopexy either by endoscopic or open surgery is the procedure of choice. Otherwise sigmoid resection with end to end anastomosis is a definite treatment of choice. In gangrenous sigmoid volvulus, Hartmann procedure with end colostomy is the safe and life saving procedure. We had a case of gangrenous sigmoid volvulus presented with pain, distension and absolute constipation with toxic symptoms. After taking X-ray erect abdomen the case was diagnosed as volvulus sigmoid. Hartmann's procedure was done after patient was resuscitated. Patient recovered well and discharged with a plan to close the colostomy after two months. We wish to present this case because it is a life threatening condition, death is certain if not treated early. We reviewed the literature regarding type of treatment since every surgeon is having his own line of approach to the problem.
\end{abstract}

Key words: Colostomy, Digital rectal examination - DRE, Gangrenous sigmoid, Hartmann's procedure and Sigmoidopexy.

\section{Introduction}

Sigmoid volvulus is a life threatening condition. It is one of the common surgical emergencies all over the world. It accounts 7-10\% of all intestinal obstructions [1, 2, and 3]. The condition is most common in African and Indian population, relatively rare in western population [3, 4]. Sigmoid volvulus is common in both sexes, but generally it is a disease of elderly male but can present in young individuals including neonates since congenitally long and narrowed mesentery is prime cause for volvulus [5].

Pain, distension of abdomen and absolute constipation is the hall mark of symptoms. The patient who presents late may have complicated gangrenous sigmoid associated with high mortality [6, 7, and 8]. X-Ray erect abdomen is diagnostic where it shows as 'bent inner tube' sign or 'coffee bean' sign. It presents in two distinct forms non gangrenous and gangrenous sigmoid volvulus. Resection and primary end to end anastomois is the treatment for non gangrenous sigmoid volvulus. Hartmann's procedure is the treatment of choice for gangrenous sigmoid volvulus.

\section{Case report}

45 year old male presented with constipation for three days, abdominal distension two days and pain one day duration. No h/o vomiting. On examination abdomen was distended and distension was increasing in size. Distension was more in the upper abdomen than the lower abdomen. Maximum tenderness was present over left ileac fossa. No rigidity or guarding was present. Bowel sounds were sluggish. No free fluid was found in the abdomen. No organomegaly. On DRE rectum was empty and was ballooned. Our provisional diagnosis was volvulus sigmoid because of asymmetrical distension of abdomen and increasing distension. Diagnosis was confirmed by plain X-ray erect abdomen was showing distended colonic loops present in the upper part of the left hypochondrium with coffee bean bent inner tube' appearance (fig.1). But usually the distended loops are present in the right hypochondriem. We suspected gangrene since patient was presenting with toxic symptoms like tachycardia, tachypnea and hypotension. 


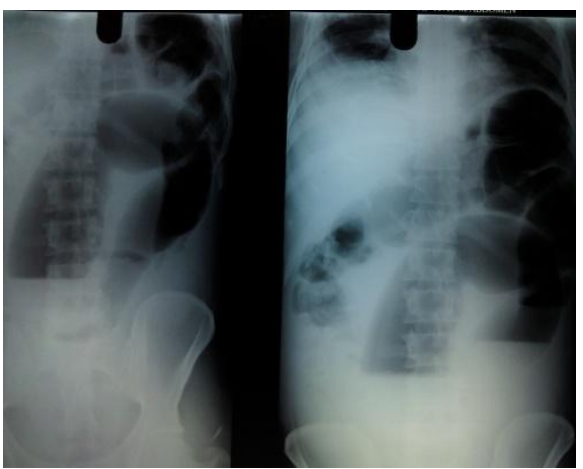

Figure 1 Erect abdomen X-ray showing distended loops of large bowel aggregated in left hypochondrium with some of the loops showing a coffee been appearance

After resuscitation under general anaesthesia abdomen was opened by midline incision. Huge dilated bluish black sigmoid colon was protruding out through the incision (fig.2). Twisted sigmoid was delivered out of abdomen and it was untwisted. Sigmoid colon was having narrow mesocolon and twisted on its narrow mesentery. Sigmoid was still bluish black in colour with thrombosed non pulsating vessels (fig.3). Sigmoidectomy was done (Hartmann's operation) with proximal end colostomy, primary end to end anastomosis was differed since sigmoid was gangrenous.

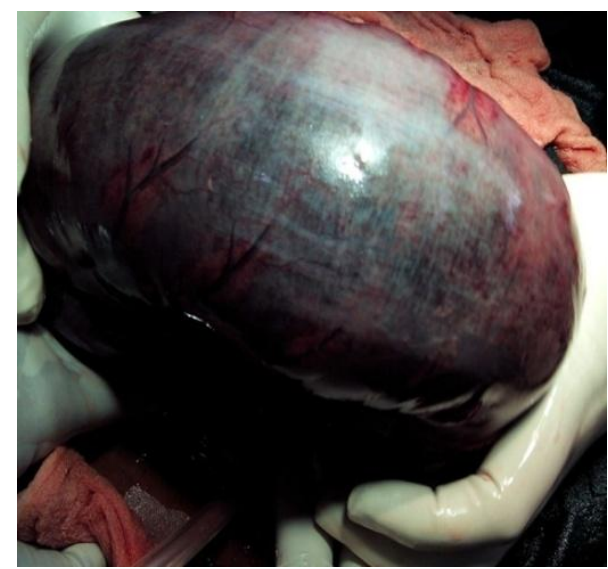

Figure 2 Distended gangrenous sigmoid colon coming out of the incision.

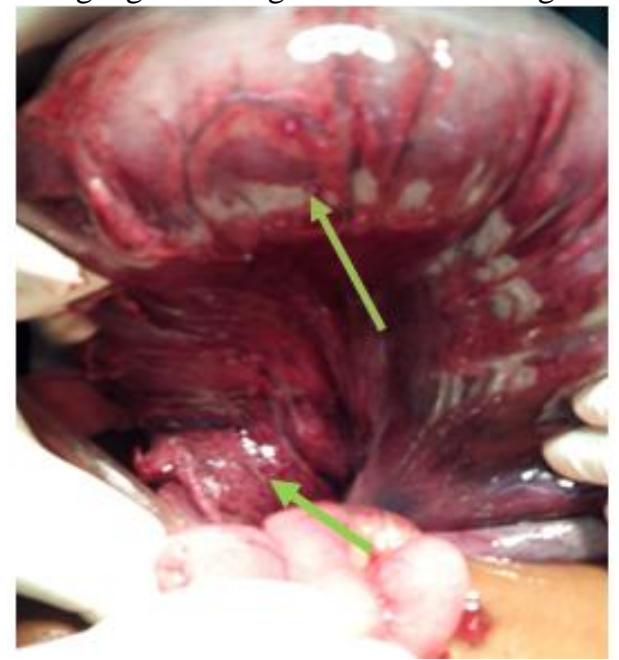

Figure 3. Showing narrow mesentery with thrombosed vessels.

\section{Discussion}

Sigmoid volvulus is a life threatening condition accounts 5-7\% of all intestinal obstruction. Sigmoid volvulus is third most common cause of large bowel obstruction and common all over the world but more common in Africa, India and east European countries [3, 4]. Generally it is disease of elderly male but females can have sigmoid volvulus as well as young individuals and neonates can get effected [5, 6, 7, and 8]. It 
associates high mortality 20-30\%. Mortality depends on time of onset and arrival to hospital. Mortality is much higher if patient presenting with gangrene of the sigmoid [6]. Mortality is still high if patient presents with perforation. Death is certain if not intervened in time.

Exact aetiology is not known. Most of the authors claim that it is an acquired condition but per operatively there is long sigmoid mesocolon with proximal narrowing which gives us a concept of congenital problem. The narrowest portion of sigmoid mesocolon is the site of torsion. The second precipitating cause is loaded sigmoid with fecal matter which encourages the segment to twist on its mesentery which gives us a concept of acquired condition. Clinically more number of patients is elderly, which supports the concept of acquired condition, but it is also seen in young individuals, infants and neonates $[7,8]$ which are in favour of congenital condition. Patient gives h/o chronic constipation and high residue diet encourages constipation. Congenital long mesocolon and acquired constipation seems to be cause for sigmoid vovulus in India rather than psychiatric problems. Long mesocolon also delays the faecal evacuation which leads to chronic constipation.

Patient presents with pain and distension of the abdomen with absolute constipation. Pain is in the lower abdomen more towards the left iliac fossa. Pain is localised, continuous and agonising. Pain can also be a colicky type from distended sigmoid. Distension is more on upper abdomen than the lower and it is a characteristic feature of sigmoid volvulus. Tenderness is present all over the abdomen but more in the left ileac fossa. Resonance note is present more on upper abdomen on percussion. Bowel sounds are high pitched but as time passes on bowel sounds are usually sluggish. Patient presents with toxic symptoms such as tachycardia, tachypnea with elevated temperature if sigmoid colon presents with gangrene.

Plain X-ray erect abdomen is diagnostic as it shows pneumatic distended loops of large bowel mostly on right upper quadrant or in left upper quadrant. In our case dilated loops were located in left upper quadrant. Loss of haustrational foldings of colon is seen. There is 'inner tube bent' sign or 'coffee bean' sign present on plain $\mathrm{x}$-ray and it is diagnostic.

Before surgery patient is resuscitated well, dehydration and serum electrolytes are corrected. Blood grouping and $\mathrm{Rh}$ typing is carried out since patient may require blood transfusion after bowel excision. Upper GI tract is decompressed with nasogastric tube. Foley's catheter is passed to assess the urine output.

There are few surgical options are available for volvulus sigmoid like resection and primary anastomosis, Hartmann's procedure, mesosigmoidopexy and endoscopic sigmoidopexy. Surgical option depends on whether the sigmoid volvulus is simple volvulus without gangrene or volvulus with gangrene.

Resection with primary anastomois is the treatment in non gangrenous sigmoid volvulus. It demands on table preparation of colon since colon is loaded with faecal matter. On table preparation contaminate the peritoneal cavity as spillage is present which leads to wound sepsis sometime peritonitis. There can be a chance of anastomotic leak due to incomplete on table preperation of colon [9]. Anastomotic leak leads to faecal peritonitis with increased morbidity and mortality. Patient has to be kept nil per oral for prolonged period.

Another procedure is detorsion followed by sigmoidopexy particularly when surgeon is inexperienced. It can be done by endoscopy or by open methods [10].This avoids peritoneal soiling and patient life is secured. But it is not a treatment of the problem rather it is relief of the problem since it is not removing the cause [11].

Treatment is differed for gangrenous sigmoid volvulus since it associates with high mortality accounting 10-40\% [7, 8]. Excision and end to end anastomosis is not entertained because it is difficult to assess normal viable bowel. If viable, wall is edematous due to accumulation of inflammatory fluid leaving more chance of stomal leakage. General condition of the patient is already in jeopardy. Keeping all these factors in view Hartmann operation is the procedure of choice i.e. resection of sigmoid volvulus with proximal loop end colostomy and distal loop is closed and left in the pelvis.

What we feel, detorsion and sigmoidopexy is not the solution for the problem. Patient is left for another surgery since volvulus invariably recurs after sometime. If resection is attempted proximal dysfunctiong colostomy is mandate since anastomotic leak associate with high morbidity and mortality. Hartmann's procedure is golden option for gangrenous sigmoid volvulus.

\section{Conclusion}

Volvulus sigmoid is relatively common surgical emergency. It is common from neonate to elderly people. It is diagnosed clinically and can be confirmed by plain radiograph. Few treatment options are available. Detorsion and primary excision of sigmoid and end to end anastomosis is the treatment of choice in non gangrenous sigmoid volvulus. Yet chance of leak is high in primary anastomosis since colon is not prepared. Hartmann procedure is golden option in gangrenous volvulus of the sigmoid.

\section{Reference}

[1]. Udezue N. Sigmoid volvulus in Kaduna, Nigeria. Diseases of the Colon and Rectum. 1990; 33:647- 649. [PubMed: 2376220]

[2]. SchagenVanLeeuwen J. Sigmoid volvulus in a West African population. Diseases of the Colon and Rectum. 1985; 28:712-716. [PubMed: 4053876] 
[3]. Lal S, Morgenstern R, Vinjirayer E, Matin A. Sigmoid volvulus an update. Gastrointestinal endoscopy clinics of North America. 2006; 16:175-187. [PubMed: 16546032]

[4]. Ballantyne G, Brandner M, Beart R, Ilstrup D. Volvulus of the colon. Incidence and mortality. Annals of Surgery. 1985; 202:8391. [PubMed: 4015215]

[5]. Valentine Pastore, Angela Basile, Raffaella Cocomazzi, Marlena Pastore, Fabio Bartoli. Sigmoid volvulus in a neonate: Case report and review of literature; African J ournal of paediatric surgery Oct/Decm 2013 (10) issue 4; 390-392.

[6]. Raveenthiran V. Restorative resection of unprepared left-colon in gangrenous versus viable sigmoid volvulus. International Journal of Colorectal Diseases. 2004; 19:258-263.

[7]. Grossmann EM, Longo WE, Stratton MD, et al. Sigmoid volvulus in Department of Veterans Affairs Medical Centers. Dis Colon Rectum 2000; 43:414-8.

[8]. Connolly S, Brannigan AE, Heffeman E, Hyland JM. Sigmoid volvulus: a 10-year-audit. Ir J Med Sci $2002 ; 171: 216-7$.

[9]. Atamanalp SS, Yildirgan MI, Basoglu M, et al. Sigmoid colon volvulus in children: review of 19 cases. Pediatr Surg Int 2004; $20: 492-5$.

[10]. Muyco A, Kushner A, Mvula C. Management of sigmoid volvulus at a tertiary care hospital in Africa. East and Central African Journal of Surgery. 2005; 10:105.

[11]. Northeast AD, Dennison AR, Lee EG. Sigmoid volvulus: new thoughts on the epidemiology. Dis Colon Rectum 1984; $27: 260-1$. 